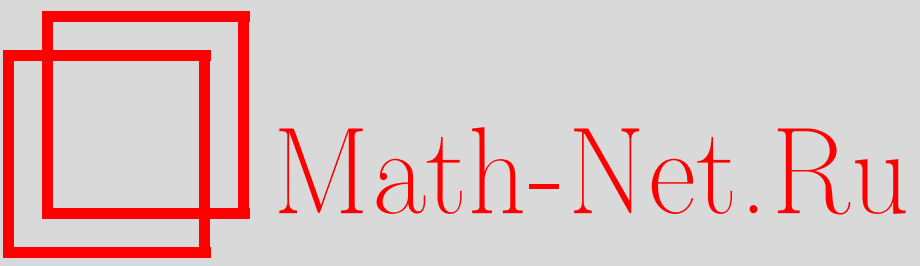

В. Р. Халилов, Электрослабые распады нуклонов в сверхсильном магнитном поле, ТМФ, 2005, том 145, номер 1, 108-122

DOI: https://doi.org/10.4213/tmf1886

Использование Общероссийского математического портала Math-Net.Ru подразумевает, что вы прочитали и согласны с пользовательским соглашением

http://www.mathnet.ru/rus/agreement

Параметры загрузки:

IP: 54.198 .55 .26

26 апреля 2023 г., 13:09:35 
ТЕОРЕТИЧЕСКАЯ

И МАТЕМАТИЧЕСКАЯ

ФИЗИКА

Том 145, № 1

октябрь, 2005

(C) 2005 г.

В.Р. Халилов*

\section{ЭЛЕКТРОСЛАБЫЕ РАСПАДЫ НУКЛОНОВ В СВЕРХСИЛЬНОМ МАГНИТНОМ ПОЛЕ}

\footnotetext{
Изучается влияние магнитного поля на электрослабые процессы распада нуклонов в вырожденном идеальном газе нейтронов, протонов и электронов, находящемся во внешнем сверхсильном постоянном и однородном магнитном поле, с учетом эффектов, обусловленных взаимодействием аномальных магнитных моментов нуклонов с магнитным полем. Получены выражения для вероятностей электрослабых процессов, которыми, как предполагается, поддерживается химическое равновесие в центральной области нейтронной звезды с вмороженным сверхсильным магнитным полем, при различных значениях химических потенциалов вырожденных фермионов. Показано, что различие вероятностей распада нейтрона в магнитном поле $B \ll 10^{17}$ Гс и в отсутствие его полностью обусловлено изменением фазового объема электронных состояний. Обсуждается распад протона на нейтрон, позитрон и нейтрино. Этот процесс энергетически разрешен только при учете взаимодействия аномальных магнитных моментов нуклонов с сверхсильным магнитным полем.
}

Ключевые слова: сильное магнитное поле, квантование уровней энергии, нейтронная звезда, электрослабые процессы.

\section{1. ВВЕДЕНИЕ}

Проблемы влияния сильного магнитного поля на поведение и свойства вешества высокой плотности при протекании в нем различных микропроцессов с участием элементарных частиц в последние годы приобретают новый интерес в связи с открытием компактных нейтронных звезд с вмороженными сверхсильными магнитными полями, названных магнитарами [1], [2]. Как предполагают [3], [4], величина напряженности магнитного поля $B$ на поверхности этих звезд может достигать значений $10^{15}$ Гс. Существование таких полей на поверхности магнитаров недавно было подтверждено из наблюдений [5]. Можно ожидать, что магнитная индукция в центре магнитара превышает ее значение на поверхности на несколько порядков. Однако существует верхний предел магнитной индукции в центре нейтронной звезды, выше которого нейтронная звезда (данной массы) становится нестабильной. Поэтому условие стабильности нейтронной

* Московский государственный университет, Москва, Россия. E-mail: khalilov@thc.phys.msu.su 
звезды накладывает вытекающее из скалярной теоремы вириала ограничение на максимально допустимое значение внутреннего магнитного поля, вмороженного в звезду [6]. При этом если напряженность магнитного поля на поверхности нейтронной звезды порядка $10^{15}$ Гс, то в центральной области звезды магнитная индукция может стать порядка $10^{18}$ Гс [7]. Сушествование вмороженного сверхсильного магнитного поля приводит к возрастанию величины максимально возможной массы стабильной нейтронной звезды по сравнению с ее значением без магнитного поля [8].

Магнитное поле оказывает существенное влияние на различные микропроцессы, которые могут протекать как вблизи поверхности магнитара, так и в сверхплотной ядерной материи в центральной области нейтронной звезды (см. [8]-[24]).

Одним из важных процессов, ведущих к образованию нейтронной звезды, является процесс нейтронизации. Реакция нейтронизации в веществе начинается при высоких плотностях, когда энергия электронов становится достаточной для обратного $\beta$-распада с преврашением протонов (как связанных в ядрах, так и свободных) в нейтроны с захватом электронов и образованием нейтрино. Впервые анализ динамики процесса захвата электрона ядром $\mathrm{He}^{4}$ с образованием трития $\mathrm{T}$, нейтрона и нейтрино $e^{-}+\mathrm{He}^{4}=$ $\mathrm{T}+n+\nu$, за которым следует реакция $e^{-}+\mathrm{T}=3 n+\nu$, был проведен в работе [16]. При коллапсе звезды до нейтронной в этих процессах образуются нейтрино больших энергий. Всесторонне процессы нейтронизации в веществе рассмотрены в обзоре [17].

Химическое равновесие в образовавшемся в центральной области нейтронной звезды вырожденном газе нуклонов и электронов $\left(n p^{+} e^{-}\right.$-газ) поддерживается процессами [8]-[11]

$$
\begin{aligned}
& n \rightarrow p^{+}+e^{-}+\tilde{\nu}, \\
& p^{+}+e^{-} \rightarrow n+\nu
\end{aligned}
$$

где $\tilde{\nu}$ и $\nu$ обозначают антинейтрино и нейтрино, соответственно. В этих процессах сохраняется плотность полного числа барионов $\left(n_{b}=n_{p}+n_{n}\right)$ и выполняется условие электронейтральности $n_{p}=n_{e}$.

Магнитары относят к сравнительно молодым нейтронным звездам. Поэтому не лишен интереса вопрос о соотношении времени, необходимого для установления химического равновесия $n p^{+} e^{-}$-газа, и времени $T_{B}$, за которое магнитное поле в ядре магнитара достигает своего равновесного значения. Время $T_{B}$ можно оценить при разумном предположении, что скорость нарастания магнитного поля по порядку величины равна скорости свободного падения частицы при гравитационном сжатии звезды до нейтронной. Тогда нетрудно получить следуюшую оценку: $T_{B} \sim R^{3 / 2} /\left(c r_{\mathrm{g}}^{1 / 2}\right)$, где $R$ - радиус коллапсирующей звезды, $r_{\mathrm{g}}=G M / c^{2}$ - так называемый гравитационный радиус, $G-$ гравитационная постоянная, $c$ - скорость света. Мы также учли, что радиус нейтронной звезды в несколько раз превышает гравитационный радиус звезды той же массы. Для звезды с массой и радиусом Солнца $T_{B} \sim 10^{3}$ c. Интересно, что это значение $T_{B}$ практически совпадает со временем свободного распада нейтрона $1013 \mathrm{c}$. 
Заметим, что характерный период движения заряженной частицы в магнитном поле (не связанный с электрослабыми процессами) $t \sim l / v$, где $l$ - линейный размер области движения частицы в магнитном поле, $v$ - характерная скорость частицы, удовлетворяет неравенству $t \ll T_{B}$, так что можно считать, что магнитное поле по отношению к указанным движениям нарастает адиабатически. Вместе с этим промежуток времени, за который вмороженное в звезду магнитное поле достигает своего равновесного (для стабильности звезды) постоянного значения, мал по сравнению с характерными временами процессов, приводящих к образованию $n p^{+} e^{-}$-газа в ядре магнитара. Поэтому при вычислении вероятностей рассматриваемых ниже электрослабых процессов в $n p^{+} e^{-}$-газе магнитное поле считается постоянным, а время релаксации $n p^{+} e^{-}$-газа, которое определяется этими электрослабыми процессами, а следовательно, и скорость охлаждения нейтронной звезды (на конечной стадии) будут существенно зависеть от концентраций частиц и величины магнитной индукции, которые установились в ядре магнитара к началу процесса релаксации.

Здесь мы рассмотрим влияние сверхсильного магнитного поля на динамику элементарных электрослабых процессов, приводящих к установлению химического равновесия в физической системе, представляюшей вырожденный идеальньй $n p^{+} e^{-}$-газ во внешнем сверхсильном постоянном и однородном магнитном поле.

\section{2. СПЕКТРЫ ЭНЕРГИЙ И ПЛОТНОСТИ КВАНТОВЫХ СОСТОЯНИЙ ФЕРМИОНОВ В МАГНИТНОМ ПОЛЕ}

В магнитном поле $B=B_{0}=m^{2} /|e|=4.4 \cdot 10^{13} \Gamma$ c $(m$ и $e<0$ - масса и заряд электрона) для электрона и $B=B_{0}^{*}=m_{p}^{2} / e=3.4 \cdot 10^{6} B_{0}=1.5 \cdot 10^{20} \Gamma$ с $\left(m_{p}\right.$ и $e>0$-масса и заряд протона) для протона существенны эффекты квантования орбитального движения для этих частиц. Экспериментальные значения аномальных магнитных моментов $(\mathrm{AMM})$ нуклонов: $M_{p}=\left(g_{p} / 2-1\right) M_{N}$ для протона, $M_{n}=g_{n} M_{N} / 2$ для нейтрона, где $M_{N}=e /\left(2 m_{p}\right)$ - ядерный магнетон, а $g_{p}=5.58, g_{n}=-3.82-g$-факторы Ланде для протона и нейтрона, соответственно. Поэтому энергия взаимодействия АММ нуклона с магнитным полем становится порядка $m c^{2}$ в поле $B \sim B_{m}=\left(m_{p} / m\right) B_{0}$. В магнитных полях $B=m_{p, n} / M_{p, n} \leqslant B_{0}^{*}$ для протона и нейтрона их энергии связи при определенных значениях проекций спинов частиц на направление магнитного поля могут стать равными энергиям покоя этих частиц. Вместе с этим следует помнить, что уравнение Дирака-Паули обосновано для нуклонов только в нерелятивистском приближении и при $B \ll B_{0}^{*}$.

Так как мы ограничимся рассмотрением эффектов в области значений $B \ll B_{0}^{*}$, для спектров энергий нуклонов используем выражения

$$
\begin{aligned}
& E_{p}=m_{p}-s_{p} M_{p} B+\frac{\mathbf{p}_{p}^{2}}{2 m_{p}}, \\
& E_{n}=m_{n}+s_{n} M_{n} B+\frac{\mathbf{p}_{n}^{2}}{2 m_{n}},
\end{aligned}
$$


где $\mathbf{p}_{p}$ и $\mathbf{p}_{n}, m_{p}$ и $m_{n}-$ импульс и масса протона и нейтрона, $s_{p, n}= \pm 1-$ квантовое число, характеризуюшее проекцию спина фермиона на направление внешнего магнитного поля.

Так как вклад АММ электрона в собственные значения энергии электрона в области значений $B \ll B_{0}^{*}$ мал, мы его не учитываем, а спектр энергий электрона во внешнем постоянном и однородном магнитном поле используем в виде (см., например, [15])

$$
E=\sqrt{m^{2}+2 k|e| B+p^{2}}
$$

где $p, s= \pm 1$ - проекции импульса и спина на направление внешнего магнитного поля, соответственно, $k=0,1,2, \ldots$ - главное квантовое число, нумерующее уровни Ландау.

Связи меж ду энергиями Ферми (или химическими потенциалами) и плотностями числа нуклонов в области значений $B \ll B_{0}^{*}$ описываются теми же формулами, что и в отсутствие магнитного поля, в которых вместо $m_{p}$ и $m_{n}$ можно использовать выражения $m_{p}^{*}=m_{p}-s_{p} M_{p} B$ и $m_{n}^{*}=m_{n}+s_{n} M_{n} B$, соответственно. Связь между энергией Ферми электрона $\mu_{e}$ в присутствии магнитного поля и плотностью числа электронов $n_{e}$ определяется выражением [13]

$$
\frac{\sqrt{2} n_{e}}{3 n_{0}}\left(\frac{B_{0}}{B}\right)^{3 / 2}=\left(\left(\mu^{2}-1\right) \frac{B_{0}}{2 B}\right)^{1 / 2}+2 \sum_{k=1}^{k_{\max }}\left(\left(\mu^{2}-1\right) \frac{B_{0}}{2 B}-k\right)^{1 / 2}
$$

где $\mu=\mu_{e} / m$, а целое число $k_{\max }$ - целая часть функции $\left(\mu^{2}-1\right) B_{0} /(2 B)$ - определяется энергией Ферми $\mu_{e}$ :

$$
k_{\max }=\left[\frac{\mu_{e}^{2}-m^{2}}{2|e B|}\right]
$$

Так как энергия Ферми в отсутствие магнитного поля $\mu_{0}$ связана с плотностью числа электронов $n_{e}$ с помощью формулы

$$
\left(\frac{\mu_{0}^{2}-m^{2}}{m^{2}}\right)^{3 / 2}=\frac{n_{e}}{n_{0}}, \quad n_{0}=\frac{m^{3}}{3 \pi^{2}}
$$

то фактически формула (5) неявно определяет энергию Ферми вырожденных релятивистских электронов в присутствии внешнего постоянного магнитного поля.

Если плотность электронов такова, что $n_{e} / n_{0} \gg\left(B / B_{0}\right)^{3 / 2}$, а $B \ll B_{0}$, то электроны заполняют много уровней Ландау, а их энергия Ферми мало отличается от соответствуюшего выражения в отсутствие поля. Однако если

$$
\frac{\sqrt{2} n_{e}}{3 n_{0}}\left(\frac{B_{0}}{B}\right)^{3 / 2}<1
$$


то только низший уровень $k=0$ может быть заполнен электронами. Неравенство (8) можно также переписать в форме

$$
\frac{n_{e}}{n_{0 n}}<\frac{3}{\sqrt{2}}\left(\frac{m^{2} B}{m_{n}^{2} B_{0}}\right)^{3 / 2} \simeq 3.4 \cdot 10^{-10}\left(\frac{B}{B_{0}}\right)^{3 / 2}, \quad n_{0 n}=\frac{m_{n}^{3}}{3 \pi^{2}}
$$

Если заполнен только уровень $k=0$, то для заданного $n_{e}$ из (5) получим соотношение

$$
\left(\mu^{2}-1\right)^{1 / 2} \equiv \frac{p_{\mathrm{F}}}{m}=\frac{2 B_{0} n_{e}}{3 B n_{0}}
$$

где $p_{\mathrm{F}}-$ импульс Ферми.

\section{3. ВЕРОЯТНОСТИ ЭЛЕКТРОСЛАБЫХ РАСПАДОВ НУКЛОНОВ}

Вероятности соответствуюших электрослабых процессов, которыми определяется скорость приближения (или время релаксации) системы к химическому равновесию, зависят от химических потенциалов частиц и магнитной индукции, значения которой считаем ограниченными интервалом $B_{0} \ll B \leqslant 10^{2} B_{m} \sim 8 \cdot 10^{18}$ Гс. Тогда в первом приближении можно пренебречь эффектами, связанными с квантованием уровней энергии для протона, и в расчетах матричных элементов использовать свободные волновые функции для всех частиц, за исключением электрона и позитрона. В поле же $B \sim B_{m}$ энергию взаимодействия АММ нуклона с магнитным полем следует учитывать в собственных значениях энергии нуклонов. Следует отметить, что распад покояшегося нейтрона в постоянном и однородном магнитном поле без учета вкладов АММ фермионов изучался в ряде работ (см., например, [18]-[22]).

Волновую функцию электрона в магнитном поле $\mathbf{B}=(0,0, B)$ в калибровке векторного потенциала $\mathbf{A}=(0, x B, 0)$ можно записать через положительно-частотные решения уравнения Дирака в следуюшем виде:

$$
\Psi(t, \mathbf{r})=\frac{1}{L} e^{-i E_{k} t+i p_{2} y+i p_{3} z}\left(\begin{array}{c}
C_{1} U_{k-1}(X) \\
i C_{2} U_{k}(X) \\
C_{3} U_{k-1}(X) \\
i C_{4} U_{k}(X)
\end{array}\right)
$$

где $E_{k}=\sqrt{E_{\perp}^{2}+p_{3}^{2}}, E_{\perp}^{2}=m^{2}+2 k|e B|$, а $L-$ линейный размер области движения электрона. Коэффициенты $C_{j}$ для конкретного сохраняюшегося в магнитном поле оператора поляризации, характеризующего проекцию спина электрона на направление магнитного поля $(s= \pm 1)$, можно представить в виде

$$
\left(\begin{array}{l}
C_{1} \\
C_{2} \\
C_{3} \\
C_{4}
\end{array}\right)=\frac{1}{2 \sqrt{2 E_{\perp} E_{k}}}\left(\begin{array}{c}
D_{1}\left(A_{1}+A_{2}\right) \\
D_{2}\left(A_{2}-A_{1}\right) \\
D_{1}\left(A_{1}-A_{2}\right) \\
D_{2}\left(A_{1}+A_{2}\right)
\end{array}\right)
$$


где

$$
\begin{array}{ll}
D_{1}=\sqrt{2 k|e| B+s m}, & D_{2}=s \sqrt{2 k|e| B-s m}, \\
A_{1}=\sqrt{E_{k}+p_{3}}, & A_{2}=s \sqrt{E_{k}-p_{3}},
\end{array}
$$

а функции

$$
U_{k}(X)=\frac{|e B|^{1 / 4}}{\left(2^{k} k ! \pi^{1 / 2}\right)^{1 / 2}} e^{-X^{2} / 4} H_{k}\left(\frac{X}{\sqrt{2}}\right)
$$

выражены через полиномы Эрмита $H_{k}(X / \sqrt{2})$ аргумента $X=\sqrt{2|e B|}\left(x-p_{2} /(e B)\right)$. Электроны в сильном магнитном поле $B \gg B_{0}$ локализованы в поперечной магнитному полю плоскости в области с линейным размером порядка $(1 / m) \sqrt{B_{0} / B} \equiv \lambda \sqrt{B_{0} / B}$ (см., например, [25]).

Нелишне отметить, что, так как при $k=0$ функция $U_{-1}(X)=0$, проекция спина электрона на направление магнитного поля принимает только одно значение $(s=-1)$, а не два, как в других состояниях. Инвариантная амплитуда вероятности перехода из начального состояния (i) в конечное состояние (f) (для определенности электрослабого распада нейтрона $n \rightarrow p^{+}+e^{-}+\bar{\nu}$ ) определяется следуюшим матричным элементом:

$$
T_{\mathrm{fi}}=\frac{G}{\sqrt{2}} \int d^{4} x\left[\bar{\Psi}_{p}(x) \gamma_{\mu}\left(1-\alpha \gamma_{5}\right) \Psi_{n}(x)\right]\left[\bar{\Psi}_{e}(x) \gamma^{\mu}\left(1-\gamma_{5}\right) \Psi_{\nu}(x)\right],
$$

где $G / \sqrt{2}=e^{2} /\left(8 m_{W}^{2} \sin ^{2} \theta_{\mathrm{W}}\right), m_{W}$ - масса $W$-бозона, $\theta_{\mathrm{W}}$ - угол Вайнберга, $\alpha$ - отношение аксиально-векторной и векторной констант слабого взаимодействия, $\Psi_{n}(x)$, $\Psi_{p}(x), \Psi_{e}(x)$ и $\Psi_{\nu}(x)$ - волновые функции нейтрона, протона, электрона и антинейтрино, соответственно, а $x \equiv x^{\mu}=(t, \mathbf{r})$ - 4-вектор. Интегрирование по $t, y$ и $z$ тривиально и приводит к произведению трех $\delta$-функций; интегрирование по $x$ выполняется с помошью формулы [26]

$$
\int_{-\infty}^{\infty} e^{i x y} U_{k}(X) d x=\sqrt{\frac{2 \pi}{|e B|}} U_{k}\left(\frac{y}{\sqrt{|e B|}}\right) e^{i k \pi / 2+i y p_{2} /|e B|},
$$

где $y=p_{1 n}-p_{1 p}-p_{1 \nu}$.

Вероятность распада нейтрона в однородном магнитном поле в единицу времени имеет следующий вид:

$$
\begin{aligned}
d w= & (2 \pi)^{3} \delta\left(E_{n}-E_{p}-E_{k}-E_{\nu}\right) \delta\left(p_{3 n}-p_{3 p}-p_{3}-p_{3 \nu}\right) \times \\
& \times \delta\left(p_{2 n}-p_{2 p}-p_{2}-p_{2 \nu}\right)\left|M_{\mathrm{fi}}\right|^{2} \prod_{f} \frac{d^{3} p_{\mathrm{F}}}{L(2 \pi)^{3}},
\end{aligned}
$$

где, с учетом сделанного в начале этого раздела замечания, энергии нейтрона и протона в аргументе $\delta$-функции имеют вид

$$
\begin{aligned}
& E_{n}=m_{n}-1.91 s_{n} M_{N} B+\frac{\mathbf{p}_{n}^{2}}{2 m_{n}}, \\
& E_{p}=m_{p}-1.79 s_{p} M_{N} B+\frac{\mathbf{p}_{p}^{2}}{2 m_{p}},
\end{aligned}
$$


a $\left|M_{\mathrm{fi}}\right|^{2}$ не содержит $\delta$-функций, длины $L$ и квантового числа $p_{2}$.

Например, вычисление полной вероятности распада нейтрона проводится следующим образом. Сначала выполняется интегрирование по проекции импульса протона $p_{1 p}$, причем, если магнитная индукция удовлетворяет условию $B \ll B_{m}$, интегрирование по $p_{1 p}$ можно проводить в бесконечных пределах с помощью интеграла

$$
\int_{-\infty}^{\infty} U_{k}^{2}\left(\frac{y}{\sqrt{|e B|}}\right) d\left(\frac{y}{\sqrt{|e B|}}\right)=1
$$

Интегрирование по импульсам антинейтрино (которое удобно проводить в сферических координатах $\left.d^{3} p_{\nu}=E_{\nu}^{2} d E_{\nu} \sin \theta_{\nu} d \phi_{\nu}\right)$ и оставшиеся интегрирования по $p_{2 p}, p_{3 p}$ проводятся с помошью трех $\delta$-функций, причем интегрирование по углам $\theta_{\nu}, \phi_{\nu}$ выполняется явно. Интегрирование по $p_{2}$ дает “фазовый объем” $|e B| L$.

В результате после тривиального суммирования и усреднения по спиновым состояниям конечных и начальных частиц, соответственно, в формуле для полной вероятности остается суммирование по квантовому (электронному) числу $k$ и интегрирование по $p_{3}$ (см. [27], где при вычислениях использовались точные решения уравнения Дирака в магнитном поле и для протона):

$$
w(B)=w_{0} \frac{B}{B_{0}} \sum_{k=0}^{k_{\max }}\left(1-\frac{\delta_{k 0}}{2}\right) \int_{0}^{z_{m}}\left(\Delta_{0}-\sqrt{z^{2}+b_{k}^{2}}\right)^{2} d z
$$

где введены обозначения

$$
\begin{gathered}
w_{0}=\frac{G^{2} m^{5}\left(1+3 \alpha^{2}\right)}{2 \pi^{3}}, \quad k_{\max }=\left[\frac{\Delta^{2}-m^{2}}{2|e B|}\right], \quad \Delta_{0}=\frac{\Delta}{m}, \quad z=\frac{p_{3}}{m}, \\
z_{m}=\sqrt{\Delta_{0}^{2}-1}, \quad b_{k}=\sqrt{1+\frac{2 B k}{B_{0}}}
\end{gathered}
$$

Замечательно, что спектр электронов, как и в распаде нейтрона в отсутствие магнитного поля, просто пропорционален фазовому объему конечных состояний в магнитном поле:

$$
\frac{E_{\perp} d E_{\perp} d p_{3}}{(2 \pi)^{2}}=\frac{|e B|}{(2 \pi)^{2}} \sum_{k=0}^{k_{\max }} d p_{3}
$$

и имеет статистическую форму, как если бы матричный элемент процесса был константой, т.е.

$T_{\mathrm{fi}} \sim(2 \pi)^{3} \sqrt{|e B|} \delta\left(E_{n}-E_{p}-E_{k}-E_{\nu}\right) \delta\left(p_{3 n}-p_{3 p}-p_{3}-p_{3 \nu}\right) \delta\left(p_{2 n}-p_{2 p}-p_{2}-p_{2 \nu}\right)$.

Поэтому в магнитном поле $B \ll B_{m}$, когда изменением фазового объема состояний протона за счет квантования уровней энергии и учета АММ протона можно пренебречь, 
различие вероятностей распада нейтрона в магнитном поле и в отсутствие поля полностью обусловлено изменением фазового объема электронных состояний.

Распад покояшегося нейтрона в присутствии внешнего магнитного поля энергетически разрешен, если $Q=m_{n}+s_{n} M_{n} B-\left(m_{p}-s_{p} M_{p} B\right)>m$. В $n p^{+} e^{-}$-среде условие, при котором нейтроны стабильны относительно прямого $\beta^{-}$-распада, требует, чтобы фермиевское море электронов было заполнено вплоть до импульса $p_{\mathrm{F}}(B)$, который превьшает максимальный импульс $p_{\max }(B)$ электрона, испускаемого в прямом $\beta^{-}$-распаде [10]. Импульс Ферми электронов, который определяется уравнением химического равновесия в магнитном поле, получен в [13], где показано, что $p_{\mathrm{F}}(B)$ имеет наименьшее значение, равное $p_{\max }(B)$, при $n_{n}=0$. Поэтому покоящийся нейтрон стабилен по отношению к прямому $\beta^{-}$-распаду в магнитном поле при любой ненулевой плотности нейтронов.

Сначала найдем полные вероятности электрослабых процессов в расчете на один нуклон, учитьвая принцип Паули только для электронов посредством функции распределения Ферми:

$$
f(E)=\frac{1}{1+e^{(E-\mu) /\left(k_{\mathrm{B}} T\right)}},
$$

где $E$ - собственные значения энергии фермиона, $\mu$ - химический потенциал, $k_{\mathrm{B}}-$ постоянная Больцмана, $T$ - температура. Распределение частиц в начальном состоянии учитывается множителем $f(E)$, в конечном состоянии - множителем $(1-f(E))$, так что принцип Паули подавляет скорости реакций через так называемый блокирующий множитель, равный доле всех незаполненных конечных состояний. При нулевой температуре для фермионов $f(E)$ представляет собой ступенчатую функцию:

$$
f(E)= \begin{cases}1, & E_{e}<\mu_{e}, \\ 0, & E_{e}>\mu_{e} .\end{cases}
$$

Из закона сохранения энергии следуют соотношения $\Delta=E_{n}-E_{p}=E-E_{\nu}$ для процесса (2) и $\Delta=E+E_{\nu}$ для процесса (1). Интегралы берутся по положительным $p_{e}, p_{\nu}$, которые допускаются этими соотношениями. Вычисленные таким образом вероятности будут также справедливы и в $n p^{+} e^{-}$-среде с относительно низкой плотностью вещества. Ниже, говоря о процессах в $n p^{+} e^{-}$-среде, мы будем иметь в виду именно такую среду.

Если плотность вырож денного электронного газа удовлетворяет неравенству (8), то все электроны могут разместиться на низшем уровне $k=0$, а так как спин электрона в основном состоянии в однородном магнитном поле может быть ориентирован только против направления магнитного поля, то вырожденный электронный газ такой плотности должен быть полностью поляризованным. Для значений магнитного поля в интервале $B_{m}>B \gg B_{0}$ предельная плотность электронов в состоянии $k=0$ составляет $n_{e} \simeq 200 n_{0}$, а энергия Ферми $\mu_{e} \simeq 24 \mathrm{MэВ;} \mathrm{если} \mathrm{же} n_{e} \gg 200 n_{0}$, а $\mu_{e} \gg 24 \mathrm{MэB}$, то электроны заполняют много уровней $k$. Энергия взаимодействия АММ нуклона с магнитным полем $B_{m}$ составляет величину порядка энергии покоя электрона (0.5 MэВ); она мала по сравнению с энергией Ферми, и в выражении для $\Delta$ ею можно пренебречь. 
В $n p^{+} e^{-}$-среде во внешнем магнитном поле $B_{m}>B \gg B_{0}$ энергетически разрешены только распады нейтронов, минимальная энергия которых удовлетворяет неравенству $E_{n}-E_{p}>\mu_{e}$. Полная вероятность распада нейтрона с энергией $E_{n}$ определяется формулой $(20)$, интегрирование в которой следует проводить в пределах от $\sqrt{\mu^{2}-b_{k}^{2}}$ до $\sqrt{\Delta_{0}^{2}-b_{k}^{2}}$, где $\mu=\mu_{e} / m$, что дает

$$
\begin{aligned}
w(B)= & 2 w_{0} \frac{B}{B_{0}} \sum_{k=0}^{k_{\max }}\left(1-\frac{\delta_{k 0}}{2}\right) b_{k}^{3}\left[\frac{z^{3}}{3}+z\left(\Delta_{k}^{2}+1-\Delta_{k} \sqrt{z^{2}+1}\right)-\right. \\
& \left.-\frac{\Delta_{k}}{b_{k}} \ln \frac{z+\sqrt{z^{2}+1}}{m}\right]{ }_{\sqrt{\mu_{k}^{2}-1}}^{\sqrt{\Delta_{k}^{2}-1}}
\end{aligned}
$$

где $\mu_{k}=\mu / b_{k}, \Delta_{k}=\Delta_{0} / b_{k}$. При низкой плотности электронов $n p^{+} e^{-}$-среды $\left(n_{e} \ll\right.$ $\left.200 n_{0}\right) \quad \mu \simeq 1+\epsilon$; испускаемые в распаде электроны будут заполнять только состояние $k=0$. В этом случае полная вероятность распада нейтрона вблизи порога стабильности $\Delta-m \ll m$ принимает вид

$$
w_{0}(B)=w_{0} \frac{16 \sqrt{2} B}{15 B_{0}}\left(\frac{\Delta-m}{m}\right)^{5 / 2}
$$

Если $E_{n}-E_{p} \gg \mu_{e}$ и $\Delta_{0}^{2} \ll 2 B / B_{0}$, то испускаемые в распаде нейтрона электроны также будут заполнять только состояние $k=0$, однако в этом случае выражение для полной вероятности процесса отлично от (24) и имеет вид

$$
w_{0}(B)=w_{0} \frac{B}{B_{0}} \Delta_{0}^{3}
$$

Если $E_{n}-E_{p} \gg \mu_{e}$ и $\Delta_{0}^{2} \gg 2 B / B_{0}$, то значения энергии испускаемых в распаде электронов достаточны, чтобы заполнялись состояния с высокими $k$. Оценку полной вероятности распада получим, выполняя в формуле (23) суммирование от $k_{\min }=\left[\left(\mu_{e}^{2}-\right.\right.$ $\left.\left.m^{2}\right) /(2|e B|)\right]$ до $k_{\max }=\left[\left(\Delta-\mu_{e}^{2}\right) /(2|e B|)\right]$, что дает

$$
\begin{aligned}
w(B) & \simeq w_{0} \Delta_{0}\left(\Delta_{0}^{2}-\mu^{2}\right)\left[\frac{\Delta_{0}^{2}}{6}+\frac{\Delta_{0}^{2}+\mu^{2}}{4}(1-\ln 2)\right] \simeq \\
& \simeq w_{0} \Delta_{0}\left(\Delta_{0}^{2}-\mu^{2}\right)\left(0.26 \Delta_{0}^{2}+0.1 \mu^{2}\right) .
\end{aligned}
$$

Видно, что в $n p^{+} e^{-}$-среде нерелятивистские нейтроны стабильны, если

$$
\Delta_{0} \simeq \frac{m_{n}}{2}\left(\frac{n_{n}}{n_{0 n}}\right)^{2 / 3}-\frac{m_{p}}{2}\left(\frac{n_{p}}{n_{0 n}}\right)^{2 / 3}<\mu, \quad \frac{n_{n}}{n_{0 n}}>\frac{n_{p}}{n_{0 n}}, \quad\left(\frac{n_{n}}{n_{0 n}}\right)^{2 / 3} \ll 1,
$$

откуда следует, что энергия Ферми электронов не должна превышать 50 МэВ. 
Полная вероятность захвата электрона с энергией $E$ протоном с энергией $E_{p}$ c образованием нейтрона с энергией $E_{n}$ в $n p^{+} e^{-}$-среде в процессе (2) определяется также формулой (23), в которой в этом случае верхние пределы интегрирования (и при необходимости суммирования) становятся нижними, а нижние, наоборот, - верхними.

Чтобы изучить рассмотренные процессы в $n p^{+} e^{-}$-среде высокой плотности, содержащей вырожденные релятивистские электроны и нерелятивистские нуклоны, нужно также учесть химические потенциалы вырожденных нуклонов. Интегрирование по энергии нуклона следует проводить с учетом функции распределения Ферми для нуклона в начальном состоянии и блокируюшего множителя для нуклона в конечном состоянии. Обозначим отнесенный к массе электрона химический потенциал вырожденных нейтронов через $\mu_{n}$, протонов $\mu_{p}$, релятивистских электронов $\mu$. Вероятность распада нейтрона в этой среде можно получить, умножая, к примеру, выражение (25) на $3 \sqrt{2}\left(m / m_{n}\right)^{3 / 2}\left(E_{n} / m\right)^{1 / 2} d E_{n} / m$ и затем интегрируя полученную формулу в пределах от $\mu_{p}$ до $\mu_{n}$.

\section{4. РАСПАДЫ ПОЛЯРИЗОВАННЫХ ЧАСТИЦ}

Рассмотрим теперь электрослабые процессы в максимально допустимом для стабильного магнитара магнитном поле $B \leqslant 10^{2} B_{m}$. Предельная плотность электронов в спин-поляризованном состоянии $k=0$ составляет $n_{e} \sim 6.4 \cdot 10^{7} n_{0}$. Эта плотность только в 1.5 раза меньше плотности электронов в состоянии химического равновесия вырожденного $n p^{+} e^{-}$-газа в центральной области звезды в отсутствие магнитного поля, где отношение $n_{e} / n_{0 n}$ составляет $\sim 0.02$. Энергия Ферми $\mu_{e} \simeq 105 \mathrm{MэВ,} \mathrm{энергия}$ взаимодействия АММ нуклона с магнитным полем $10^{2} B_{m}$ равна $30 \mathrm{MэB}$; последняя все же мала по сравнению с энергией Ферми электронов. Если плотность электронов близка к предельной для поля $B \leqslant 10^{2} B_{m}$, то нейтроны стабильны относительно прямого $\beta^{-}$-распада при $E_{n}-E_{p}<105 \mathrm{MэB.}$

Нейтроны же рождаются в процессе захвата протоном электрона из фермиевского моря в магнитном поле с вероятностью

$$
w_{0}(B)=w_{0} \frac{B}{B_{0}} \mu^{3} .
$$

Заметим, что энергия Ферми релятивистских электронов $\mu=105$ МэВ равна энергии покоя $\mu^{-}$-мезона, поэтому электронам, находяшимся вблизи уровня Ферми, становится энергетически вьгодно (при столкновениях) преврашаться в $\mu^{-}$-мезоны и в покидающие звезду пары нейтрино-антинейтрино. Следовательно, в идеальном газе нуклонов и электронов может появиться некоторое число $\mu^{-}$-мезонов. Напомним, что если энергия Ферми электронов больше 53 МэВ, то распад

$$
\mu^{-} \rightarrow e^{-}+\nu_{\mu}+\tilde{\nu}_{e}
$$

запрещен принципом Паули и $\mu^{-}$-мезон стабилен в такой среде. 
В сверхсильном постоянном и однородном магнитном поле полностью поляризован и вырожденный газ нуклонов [15]. Так, нейтроны плотности $n_{n}$ будут полностью поляризованными по спину в магнитном поле $B$, если выполняется неравенство

$$
\left(\frac{n_{n}}{n_{0 n}}\right)^{2 / 3}<\frac{2^{1 / 3}\left|M_{n}\right| B}{m_{n}} \simeq 2^{1 / 3} \frac{B}{B_{0}^{*}}
$$

Например, при ядерных плотностях нейтроны должны стать полностью поляризованными в магнитном поле $B \simeq 10^{-2} B_{0}^{*} \equiv 2.3 \cdot 10^{18} \Gamma$ с, но при более низких плотностях эффект возникает в менее сильном поле. Аналогично все протоны в магнитном поле $B_{0}^{*} \gg B \gg B_{0}$ должны также стать полностью поляризованными за счет включения взаимодействия АММ протона с магнитным полем [15]. Спиновые эффекты в электрослабых процессах в этом случае также актуальны. Прямой $\beta^{-}$-распад нейтрона в сверхсильном магнитном поле все еше энергетически разрешен даже с учетом того, что взаимодействие с полем АММ нейтрона, проекция спина которого на направление поля $s_{n}=1$, понижает энергию покоя нейтрона, при условии

$$
Q=m_{n}+s_{n} M_{n} B+\frac{m_{n}}{2}\left(\frac{n_{n}}{n_{0 n}}\right)^{2 / 3}-\left[m_{n}+s_{n} M_{n} B+\frac{m_{n}}{2}\left(\frac{n_{n}}{n_{0 n}}\right)^{2 / 3}\right]>\mu_{e}
$$

При низких плотностях

$$
\frac{n_{n}}{n_{0 n}} \simeq 10^{-6}, \quad \frac{n_{p}}{n_{0 n}}, \frac{n_{e}}{n_{0 n}} \simeq 10^{-8}, \quad \mu_{e} \simeq m
$$

нейтроны с энергией $E_{n}=m_{n}-1.91 M_{N} B$ и проекцией спина $s_{n}=1$ в магнитном поле

$$
B<\frac{m_{n}-m_{p}-m}{0.12 M_{N}} \cong 2 \cdot 10^{18} \Gamma \mathrm{c}
$$

все еше нестабильны относительно распада на протон с $E_{p}=m_{p}-1.79 M_{N} B, s_{p}=1$, электрон в состоянии $k=0, s=-1$ и антинейтрино.

При вычислении полной вероятности распада в системе отсчета, в которой нейтрон покоится в поле $B_{0}^{*} \gg B \gg B_{m}$, необходимо учесть, что электрон может рождаться только на основном уровне, а из закона сохранения энергии следует ограничение на максимально допустимую проекцию импульса протона $p_{1 p, \max }<\sqrt{2 m_{p}(Q-m)}$. Интегрирование по $p_{1 p}$ теперь тривиально и дает $2 p_{1 p \text {, max }}$ В результате выражение для вероятности распада нейтрона с образованием электрона в состоянии $k=0$, например (24), будет содержать дополнительный множитель $\sqrt{8 m_{p}(Q-m) /(|e| B \pi)}$, что дает

$$
w(B)=\frac{G^{2} m^{5}(1-\alpha)^{2}}{\sqrt{8 \pi} \pi^{3}} \sqrt{\frac{B m_{p}}{B_{0} m}}\left(\frac{Q_{0}-m}{m}\right)^{3},
$$

где $Q_{0}=m_{n}-1.91 M_{N} B-\left(m_{p}-1.79 M_{N} B\right)$. Отметим, что если при выгислении вероятности распада нейтрона использовать не волновые функции свободного протона, а 
точные решения уравнения Дирака в магнитном поле, аналогичные электронным функциям (11), то вместо интегрирования по проекции импульса протона $p_{1 p}$ в выражении для вероятности появится суммирование по дискретному квантовому числу $k^{\prime}$, обозначающему номер уровня Ландау для протона. В частности, в системе отсчета, в которой нейтрон покоится в поле $B_{0}^{*} \gg B \gg B_{m}$, суммирования по $k^{\prime}$ нет, так как из закона сохранения энергии следует, что протон может находиться только в состоянии $k^{\prime}=0$. Для получения правильного результата в этом случае необходимо учитывать неопределенность энергии протона в состоянии $k^{\prime}=0$, обусловленную нулевыми колебаниями протона в магнитном поле.

\section{5. РАСПАД ПРОТОНА В УЛЬТРАСИЛЬНОМ МАГНИТНОМ ПОЛЕ}

В центральной области магнитара протоны могут стать нестабильными относительно электрослабого позитронного $\beta^{+}$-распада

$$
p^{+} \rightarrow n+e^{+}+\nu
$$

который становится энергетически разрешенным при учете взаимодействия АММ спинполяризованных нуклонов с магнитным полем. За счет этого взаимодействия энергия протона с определенной проекцией спина может стать больше энергии нейтрона. Здесь мы рассмотрим случай, когда нейтроны могут рождаться только в энергетически низшем спин-поляризованном состоянии.

Процесс позитронного $\beta^{+}$-распада протона на нейтрон с энергией $E_{n, s_{n}=1}=m_{n}-$ $1.91 M_{N} B$, позитрон в состоянии $k=0, s=1$ и нейтрино энергетически разрешен, если $E_{p}-E_{n, s_{n}=1}-m>0$. При относительно невысоких плотностях нуклонов и электронов распад протона из состояния с энергией $E_{p, k=0, s_{p}=-1}=m_{p}+M_{p} B$ энергетически разрешен при

$$
B>\frac{m_{n}+m-m_{p}}{3.7 M_{N}} \equiv B_{p 1} \simeq 0.15 \cdot 10^{18} \Gamma \mathrm{c}
$$

с энергией $E_{p, k=1, s_{p}=1}=m_{p}+\left(2 M_{N}-M_{p}\right) B$ при

$$
B>\frac{m_{n}+m-m_{p}}{2.1 M_{N}} \equiv B_{p 2} \simeq 0.27 \cdot 10^{18} \Gamma \mathrm{c}
$$

и с энергией $E_{p, k=0, s_{p}=1}=m_{p}-M_{p} B$ при

$$
B>\frac{m_{n}+m-m_{p}}{0.12 M_{N}} \equiv B_{p 3} \simeq 4.7 \cdot 10^{18} \Gamma \mathrm{c} .
$$

В поле $B>4.7 \cdot 10^{18}$ Гс протон с $E_{p}=m_{p}-1.79 M_{N} B, s_{p}=1$ должен распадаться согласно (27) на нейтрон с $E_{n, s_{n}=1}$, позитрон в состоянии $k=0, s=1$ и нейтрино с вероятностью

$$
w(B)=\frac{G^{2} m^{5}(1-\alpha)^{2}}{\sqrt{8 \pi} \pi^{3}} \sqrt{\frac{B m_{n}}{B_{0} m}}\left(\frac{\left|Q_{0}\right|-m}{m}\right)^{3} .
$$


Заметим, что эта формула для вероятности процесса в сверхсильном магнитном поле с участием полностью спин-поляризованных массивных фермионов, в отличие от вероятностей для неполяризованных частиц, содержит множитель $(1-\alpha)^{2}$. Этот не очень сушественньй результат для электрослабых процессов с участием нуклонов (когда $\alpha \simeq$ 1.25 ) может оказаться чрезвычайно важным для электрослабых распадов кварков, обладающих АММ, вблизи порога реакции в магнитном поле $m_{q}^{2} /\left|e_{q}\right| \gg B \gg B_{0}$, где $m_{q}-$ масса, $e_{q}$ - электрический заряд кварка. Действительно, в инвариантной амплитуде вероятности перехода (14), описывающей электрослабые распады кварков, коэффициент $\alpha$ равен единице, и, следовательно, электрослабый процесс с участием массивных кварков с соответствующими проекциями спинов может стать абсолютно запрешенным в присутствии сильного магнитного поля. Нелишне отметить, что для легких кварков $\left(m_{q} \sim 10 \mathrm{MэB}\right)$ этот процесс запрещен только при $B \simeq 10 B_{0}$.

$\mathrm{B} n p^{+} e^{-}$-среде распад протона возможен лишш при определенных плотностях нуклонов. Так, нетрудно установить, что позитронный распад протона с образованием нейтрона с энергией $E_{n, s_{n}=1}$ разрешен в поле $B \geqslant B_{p 3}$ из состояния с энергией $m_{p}-M_{p} B$, если нормированные плотности нуклонов со спином $s=1$ удовлетворяют условию

$$
\frac{n_{p}}{n_{0 n}}>\frac{n_{n}}{n_{0 n}}<4.2 \cdot 10^{-2}
$$

в поле $B \geqslant B_{p 2}$ из состояния с энергией $m_{p}+\left(2 M_{N}-M_{p}\right) B$, если

$$
\frac{n_{p}}{n_{0 n}}>\frac{n_{n}}{n_{0 n}}, \quad \frac{n_{p}}{n_{0 n}}>2.2 \cdot 10^{-4}, \quad \frac{n_{n}}{n_{0 n}}<5.9 \cdot 10^{-4}
$$

и в поле $B \geqslant B_{p 1}$ из состояния с энергией $m_{p}+M_{p} B$, если

$$
\frac{n_{p}}{n_{0 n}}>2.3 \cdot 10^{-4}, \quad \frac{n_{n}}{n_{0 n}}<2.5 \cdot 10^{-4}
$$

Рож даюшихся позитронов в процессе $\beta^{+}$-распада протонов слишком мало, чтобы повлиять на концентрации фермионов в центральной области сильно намагниченной нейтронной звезды и на состояние химического равновесия $n p^{+} e^{-}$-газа, однако появление даже относительно малого количества позитронов приведет к процессу аннигиляции $e^{-} e^{+}$-пар, а также к реакции

$$
n+e^{+} \rightarrow p^{+}+\tilde{\nu}
$$

а эти процессы могут оказать влияние на процесс нейтронизации и, следовательно, на скорость охлаждения нейтронной звезды на конечной стадии ее образования.

Следует подчеркнуть, что модель Ферми с локальным четырехфермионным взаимодействием, с помошью которой в настояшей работе проводились расчеты вероятностей электрослабых процессов, перестает быть справедливой при ультравысоких плотностях среды. Так, если плотность нейтронов достигает значений, при которых энергия 
Ферми электронов становится равной по порядку величины энергии покоя промежуточного векторного $W^{-}$-бозона $\mu_{e} \simeq 100$ ГэВ, то амплитуды вероятностей электрослабых процессов следует вычислять по диаграммам калибровочной модели Вайнберга-Салама. При расчетах соответствующих диаграмм с промежуточным векторным $W^{-}$-бозоном электрослабые взаимодействия эффективно происходят в области, линейньй размер которой по порядку величины равен комптоновской длине волны $W^{-}$-бозона $\lambda_{W} \sim 10^{-16}$ см. Диаграммы с промежуточным векторным $W^{-}$-бозоном становятся актуальными при $\mu_{e} \simeq 100$ ГэВ, что соответствует плотности электронов $\sim 10^{47} \mathrm{~cm}^{-3}$. При такой чудовишной плотности область точечного взаимодействия эффективно размазывается и имеет порядок $10^{-16}$ см, а электронам, находящимся вблизи уровня Ферми, становится выгодно превращаться в $W^{-}$-бозоны и в покидающие звезду нейтрино. Таким образом, в ультраплотной среде возможно открытие новых каналов реакций.

Максимально возможные размеры области, в которой находятся электроны среды, в плоскости, перпендикулярной вектору $\mathbf{B}$, определяются величиной магнитного поля, а вдоль магнитного поля - плотностью электронов и при рассматриваемых значениях этих величин по порядку величины равны соответственно

$$
l_{\perp} \sim \sqrt{\frac{B_{0}}{B m^{2}}} \simeq 10^{-13} \mathrm{~cm}, \quad l_{\|} \sim\left(n_{e}\right)^{-1 / 3} \sim 10^{-12}-10^{-13} \mathrm{~cm} .
$$

Промежуток времени $\Delta t$, в течение которого электроны среды будут находиться в области размазывания точечного взаимодействия, очень мал по сравнению с характерными периодами их движений $T_{\perp}, T_{\|}$. Таким образом, при максимально возможных рассматриваемых значениях индукции магнитного поля и плотности электронов, полагая также, что $T_{\perp} \sim T_{\|}$, находим $\Delta t \sim 10^{-9} T_{\perp}$.

Более того, в ультраплотной среде (при $\mu_{e} \simeq 300$ ГэВ) эффективная константа слабого взаимодействия $G \mu_{e}^{2}$ возрастает до масштабов константы сильного взаимодействия, и эффекты нелокальности и вклады высших порядков начинают играть решаюшую роль.

Следовательно, расчеты вероятностей электрослабых процессов в ультраплотной среде (при $\mu_{e} \geqslant 100$ ГэВ) следует проводить в рамках калибровочной модели Вайнберга-Салама.

Благодарности. В заключение автор выражает благодарность А.Е. Шабаду за ценные замечания. Работа частично финансировалась Президентской программой поддержки ведуших научных школ (грант НШ-2027.2003.2).

\section{Список литературы}

[1] B. Paczynski. Acta Astron. 1992. V. 42. P. 145; C. Thompson, R. C. Duncan. Astrophys. J. 1996. V. 473. P. 322.

[2] R. C. Duncan. Physics in ultra-strong magnetic fields. astro-ph/0002442.

[3] C. Kouveliotou, S. Dieters, T. Strohmayer et al. Nature. 1998. V. 393. P. 235.

[4] K. Hurley, T. Cline, E. Mazets et al. Nature. 1999. V. 397. P. 41.

[5] S. Mereghetti, L. Stella. Astrophys. J. Lett. 1995. V. 17. P. L442. 
[6] C. Шапиро, С. Тьюколски. Черные дыры, белые карлики и нейтронные звезды. М.: Мир, 1985.

[7] Sut. Ghosh, San. Ghosh, K. Goswami, S. Chakrabarty, A. Goyal. Electrical conductivity at the core of a magnetar. astro-ph/0106153.

[8] D. Lai, S. Shapiro. Astrophys. J. 1991. V. 383. P. 745.

[9] R. Kippenhahn, A. Weigert. Stellar Structure and Evolution. Berlin-Heidelberg: Springer, 1994.

[10] C. Вайнберг. Гравитация и космология. М.: Мир, 1975.

[11] J. M. Lattimer, C. J. Pethick, M. Prakash, P. Haensel. Phys. Rev. Lett. 1991. V. 66. P. 2701.

[12] V. Canuto, H.-Y. Chiu. Phys. Rev. 1968. V. 173. P. 1210; P. 1220; P. 1229.

[13] В. Р. Халилов. Электроны в сильном магнитном поле. М.: Энергоатомиздат, 1988.

[14] A. Broderick, M. Prakash, J. M. Lattimer. Astrophys. J. 2000. V. 537. P. 351.

[15] В.Р. Халилов. ТМФ. 2002. Т. 130. С. 87; Т. 133. С. 103.

[16] О. Х. Гусейнов, Я. Б. Зельдович. Письма в ЖЭТФ. 1965. Т. 1. С. 11.

[17] V.S. Imshennik, D. K. Nadezhin. The terminal phases of stellar evolution in the supernova phenomenon. In: Astrophysics and Space Physics. Reviews. Sec. E. Sov. Sci. Rev. V. 2. Ed. R. A. Syunyaev. Glasgow: Bell and Bain, 1983. P. 75.

[18] Л. И. Коровина. Изв. вузов. Физика. 1964. № 6. С. 86.

[19] И. М. Тернов, Б. А. Льсов, Л.И. Коровина. Вестн. МГУ. Физика. Астрономия. 1965. № 5. C. 58.

[20] J. J. Mates, R.F. O'Connel. Phys. Rev. 1969. V. 180. P. 1289.

[21] L. Fassio-Canuto. Phys. Rev. 1969. V. 187. P. 2141.

[22] О.Ф. Дорофеев, В.Н. Родионов, И. М. Тернов. Письма в ЖЭТФ. 1984. Т. 40. С. 159; 1985. T. 42. C. 222.

[23] V.R. Khalilov. Phys. Rev. D. 2002. V. 65. P. 056001.

[24] V.I. Denisov, S. I. Svertilov. Astron. Astrophys. 2003. V. 39. P. L399.

[25] I. M. Ternov, O.F. Dorofeev, V.N. Rodionov, V. G. Bagrov, V.R. Khalilov. J. Phys. A. 1978. V. 11. P. 739; V.R. Khalilov. Electrons in Strong Electromagnetic Fields. Amsterdam: Gordon and Breach, 1996.

[26] И. С. Градитейн, И. М. Рыжик. Таблицы интегралов, сумм, рядов и произведений. М.: ГИФМЛ, 1963.

[27] А. И. Студеникин. ЯФ. 1989. Т. 49. С. 1665; В. Н. Родионов. ЖЭТФ. 1997. Т. 111. С. 3.

Поступила в редакцию 20.I.2005 г., после доработки 31.III.2005 г. 